\title{
FOCUS ON ACTIVATION, REGULATION, AND EVOLUTION OF MTI AND ETI
}

\section{MPMI Focus Issue Overview}

Ground-breaking research over recent decades has established that plants perceive a variety of molecules produced by microbes, insects, and nematodes. These pathogen-derived components include so-called microbe-associated molecular patterns, or MAMPs, as well as effector proteins that are secreted to the exterior or interior of plant cells. These molecules can be recognized by receptor protein complexes on the exterior or interior of plant cells, thereby activating MAMP- or effector-triggered immunity (MTI or ETI, respectively).

Because these processes are key components of plant disease resistance, they have been studied intensively. We are now in a golden age of ETI and MTI research, in which mechanistic and evolutionary understanding of both processes is emerging rapidly. Accordingly, the research articles and review/perspective pieces in this issue explore diverse aspects of MTI and ETI. However, a clearly apparent unifying theme for the issue is integration at multiple levels. At the cellular level, several invited reviews focus on regulatory hubs that integrate input from MTI, ETI, and other stimuli. For example, cytoskeletal arrays provide a platform for many fundamental cellular processes. $\mathrm{Li}$ and Day summarize evidence for key roles of the cytoskeleton in immune-related cellular trafficking and organization of signaling-competent microenvironments. Moreover, the cytoskeleton is targeted by pathogen effectors and, thus, mediates the balance between immunity and susceptibility (see the cover image). Calcium mediated signaling is another example of a fundamental cellular process that has been linked to many critical signaling pathways. In plants, calcium signals can be mediated by calcium-dependent protein kinases. Bredow and Monaghan provide a thorough review of the roles of calciumdependent protein kinases in plant immune signaling and highlight the challenges in understanding how this family of proteins functions at the nexus of varied inputs. The RIN4 protein is an evolutionarily conserved integrator of MTI and ETI. A review by Toruño et al. summarizes research on this intensively studied but enigmatic protein and proposes a model in which RIN4 acts as a scaffold for dynamic assembly of signaling complexes. This article, along with Li and Day's review on the cytoskeleton, draws attention to plasma membrane subdomains as critically important sites of organization for plant immune signaling.

Integration is inherent in systems-level approaches to understanding plant-microbe interactions. Mishra and Mukhtar summarize the state of the art in systems biology approaches and propose a hybridization of machine learning and network biology, with potential broad applicability. Berrabah and coauthors integrate the topics of immunity and symbiosis by summarizing our current understanding of how immune responses are manipulated to accommodate mutualistic bacteria. Key mechanisms include avoidance of surveillance and suppression of immune signaling by the bacteria and modulation of immune signaling by legume host plants. Finally, Zhu and colleagues review evidence that the agronomic benefits of intercropping arise in part from direct and indirect effects of plant immune responses, thus illustrating how a systems perspective can inform disease control strategies.

The research articles in this issue also illustrate integrative themes. For example, Cecchini and coauthors provide evidence that the signaling molecule azelaic acid induces immunity in roots through a mechanism different than previously described in shoots, and they close the paper with an intriguing invocation of the Darwin root-brain hypothesis to integrate immune regulation in the root and shoot. An example of methodological integration is provided by Maekawa and coworkers, who combine comparative genomics and functional assays for new insights into the evolution and function of barley NLR genes for powdery mildew resistance. Thomas and coworkers bring together ecological and molecular approaches, to better understand the relationship between pathogen life history traits and effectiveness of induced resistance, with implications for optimizing biocontrol strategies. Biocontrol is also the focus of paper by Agostini et al., who provide evidence that salicylic acid, jasmonic acid, and steroid signals interact during Trichoderma-induced systemic resistance in maize silks against mycotoxigenic Fusarium spp. Wang et al. use Arabidopsis genetics to identify two new negative regulators of immunity. Intriguingly, loss-of-function mutants in both genes exhibit enhanced immunity but no obvious developmental defects. Both genes are evolutionarily conserved and therefore represent promising candidates for engineered resistance via gene editing. Finally, the Pedley and Graham groups describe an NLR (nucleotide-binding site-leucine-rich repeat receptor) gene for soybean rust resistance with a novel ubiquitin protease domain that might function as an integrated decoy. This work is also an important step toward integration of foundational and translational research, because reliable sources of resistance for soybean rust are urgently needed.

We are very pleased to have assembled an issue that highlights some of the best current research in this rapidly advancing area, and we thank the authors for their splendid contributions. Unfortunately, we could not publish all of the accepted papers in the January 2019 issue. However, we look forward to seeing these papers in upcoming issues of MPMI. Topics include regulation of the oxidative burst and the impact of TCP transcription factors that represent additional hubs of integration in the plant immune network. Finally, we are laying the groundwork for the next Focus Issue on viruses, led by Senior Editors Aiming Wang and Yi Li, for publication in January 2020.

MPMI Editor-in-Chief John M. McDowell 